\title{
Phase transformation in Mn-doped titania hollow spheres and their biocompatibility studies
}

\author{
Himani Kalita - Suraj Konar · Sangeeta Tantubay • \\ Madhusudan Kr. Mahto • Amita Pathak
}

Received: 13 November 2014/ Accepted: 19 January 2015/Published online: 31 January 2015

(C) The Author(s) 2015. This article is published with open access at Springerlink.com

\begin{abstract}
Mn-doped titania hollow nanospheres were prepared via sacrificial core templating method at room temperature, using carbon spheres as the sacrificial core and template. X-ray diffraction and thermal studies showed the phase transformation of titania from anatase to rutile at temperature as low as $550^{\circ} \mathrm{C}$, when the dopant (i.e., $\mathrm{Mn}$ ) concentration was increased from 1 to $6 \mathrm{~mol} \%$ (with respect to Ti). Fourier transform infra red spectroscopic studies have been carried out to determine the surface functional groups, while the spherical and hollow morphology of the titania nanostructures have been confirmed through scanning electron microscopic as well as transmission electron microscopic studies. The chemical composition of the samples has been determined through X-ray photoelectron spectroscopic studies, while their magnetic properties have been studied using superconducting quantum interference device analysis. The biocompatibility and suitability of the nanospheres for intracellular applications has been tested through conventional MTT assay using MDA-MB 231 human breast cancer cell lines.
\end{abstract}

\footnotetext{
H. Kalita $\cdot$ S. Konar $\cdot$ S. Tantubay $\cdot$ M. Kr. Mahto .

A. Pathak $(\square)$

Department of Chemistry, Indian Institute of Technology

Kharagpur, Kharagpur 721302, West Bengal, India

e-mail: ami@chem.iitkgp.ernet.in

H. Kalita

e-mail: hkalita74@gmail.com

S. Konar

e-mail: suraj.konar@gmail.com

S. Tantubay

e-mail: sang.chem2@gmail.com

M. Kr. Mahto

e-mail: mahtomk0@gmail.com
}

Keywords Mn-doped - Titania · Hollow spheres · Anatase $\cdot$ Rutile $\cdot$ Biocompatibility

\section{Introduction}

Recently, there has been a surge of research interest in developing appropriate inorganic nanostructures for different biomedical applications. Various nanostructures of hydroxyapatite (Andronescu et al. 2013; Venkatesan et al. 2011; Wang et al. 2009), magnetite (Daumann et al. 2014; Dorniani et al. 2013), zirconia (Batra et al. 2013; Tang et al. 2010), titania (Hou et al. 2014; Aw et al. 2012), silica (Bernal et al. 2014; Xu et al. 2013) etc., have already gained attention due to their efficacy in the fabrication of biosensors and nanocarriers for targeted delivery of drugs as well as substrates for immobilization of biomolecules. Among the various inorganic materials, titania $\left(\mathrm{TiO}_{2}\right)$ holds great promise in biomedical applications owing to its wide availability, biological as well as chemical inertness and have so far been reported in various morphological forms such as nanoparticles (Liang et al. 2013), nanorods (Pang et al. 2013), nanowires (Sun and Wu 2013), nanosheets (Liu et al. 2013), nanotubes (Pandikumar and Ramaraj 2013), mesopores (Mascolo 2013), and hollow spheres (Zhao and Middelberg 2013). However, all literature reports on biomedical applications using titania based nanomaterials have been performed using titania $\left(\mathrm{TiO}_{2}\right)$ films (Kim et al. 2010), mesoporous nanoparticles (Wu et al. 2011), and nanotubes (Aw et al. 2012; Gulati et al. 2012). The hollow nanostructured titania (or, doped titania), which have the potential to create a whole new generation in host-guest chemistry because of their low density, high surface area, tunable pore structure and good surface 
permeability, have not been much explored for biomedical applications till date, according to the best of our knowledge. In an attempt to contribute in this area, we report a novel methodology for the synthesis of hollow nanospheres of $\mathrm{TiO}_{2}$ which have been tested for their biocompatibility.

From literature reports, it is evident that titania nanostructures can either be functionalized or new properties can be introduced by doping the host material with appropriate dopants. For instance, Ao et al. (2010) investigated the photocatalytic activity of N-doped titania hollow spheres for degradation of reactive Brilliant Red dye $\mathrm{X}-3 \mathrm{~B}$. Xu et al. (2006) investigated the ferromagnetic property evoked in Mn-doped $\mathrm{TiO}_{2}$ thin films and related the structural phase transition of $\mathrm{TiO}_{2}$ to the annealing temperature of the films, while Saponjic et al. (2006) studied the effect of Mn-dopant on the charge separation and surface reconstruction in Mn-doped titania nanoparticles. In the present study, titania hollow spheres have also been doped with $\mathrm{Mn}$ (II) to induce magnetic property in the material. The magnetic character developed on doping with manganese is expected to enable the magnetic field induced navigation of the titania nanospheres during biomedical application such as in targeted delivery of drugs. Further, cytotoxicity studies of the synthesized Mn-doped hollow nanospheres of $\mathrm{TiO}_{2}$ have been performed by conventional MTT assay using MDA-MB 231 cells for testing their biocompatibility.

The hollow nanospheres of titania have been prepared under ambient condition by sacrificial core templating method, where carbon spheres were used as the sacrificial core templates, while samples with different doped-Mn concentrations were obtained by varying the mole percent of $\mathrm{Mn}$ (i.e., 1, 2, 4, and $6 \mathrm{~mol} \%$ with respect to Ti). The Mn-doped hollow nanospheres of $\mathrm{TiO}_{2}$, obtained on calcination of the respective precursors at $550{ }^{\circ} \mathrm{C}$, have been characterized in detail.

\section{Experimental}

\section{Chemicals and materials}

Titanium tetraisopropoxide was purchased from Spectrochem Pvt Ltd (India), while manganese(II) acetate tetra hydrate and anhydrous dextrose (GR) were purchased from Merck (India). 3-(4, 5-dimethylthiazol-2-yl)-2,5-diphenyltetrazolium bromide (MTT) was obtained from SigmaAldrich. Human breast cancer cell lines (MDA-MB-231) were obtained from the National Center for Cell Science (NCCS), Pune (India). Absolute ethanol and milli-Q water were used throughout the study.
Preparation of carbon spheres

A $0.7 \mathrm{M}$ aqueous solution of dextrose was hydrothermally treated at $170{ }^{\circ} \mathrm{C}$ for $6 \mathrm{~h}$ in a Teflon lined autoclave under autogenous pressure. The blackish residue obtained was centrifuged, washed with water and ethanol and finally dried at $60{ }^{\circ} \mathrm{C}$ for $12 \mathrm{~h}$ to obtain the carbon spheres.

Preparation of hollow Mn-doped titania nanospheres

Initially, undoped titania hollow spheres were prepared as follows: $20 \mathrm{mmol}$ of titanium tetraisopropoxide was added to $40 \mathrm{~mL}$ ethanol. This was then mixed with $0.08 \mathrm{~g}$ of freshly prepared carbon spheres and the mixture was sonicated for $30 \mathrm{~min}$. After $6 \mathrm{~h}$ of undisturbed standing, the solution mixture was centrifuged and the collected solids were washed with ethanol, dried at $60{ }^{\circ} \mathrm{C}$ for $12 \mathrm{~h}$, and finally calcined at $550{ }^{\circ} \mathrm{C}$ for $4 \mathrm{~h}$ in air. These samples have been named as HT in the text. For preparing the Mndoped nanospheres, the procedure was repeated with the addition of the required amount of manganese(II) acetate tetra hydrate in the starting solution mixture of titanium tetraisopropoxide and carbon spheres. Four different samples with different dopant (i.e., Mn) concentrations were prepared by changing the mole percentage of $\mathrm{Mn}$ (i.e., 1, 2, 4 , and $6 \mathrm{~mol} \%$ with respect to $\mathrm{Ti}$ ) in the samples. The dried Mn-doped titania samples, containing 1, 2, 4 and $6 \%$ mole percent of $\mathrm{Mn}$ were calcined at $550{ }^{\circ} \mathrm{C}$ for $4 \mathrm{~h}$ in air and were indexed as HMT-1, HMT-2, HMT-4, and HMT6 , respectively, in the text.

Procedure for the cytotoxicity studies

The cytotoxicity of the prepared titania nanospheres were determined through conventional MTT [3-(4, 5-dimethylthiazol-2-yl)-2,5-diphenyltetrazolium bromide] assay. MDA-MB 231 cells were seeded in 96-well plate at a density of 2,000 cells per well in DMEM (Dulbecco's Modified Eagle's medium) complete medium. The cells were incubated for $16 \mathrm{~h}$ for adhesion and growth. After incubation, the complete DMEM medium was replaced by incomplete DMEM medium and the cells were treated with different concentrations of the prepared hollow nanospheres. For comparison, control wells were treated with only DMEM medium. After $48 \mathrm{~h}$ of incubation, the medium (containing the hollow nanospheres) were discarded, replaced with $100 \mu \mathrm{L}$ of MTT solution $(1 \mathrm{mg} / \mathrm{mL})$ and further incubated for $4 \mathrm{~h}$ for the reduction of MTT to formazan crystals by the viable cells. The unreduced MTT solution was then discarded and $100 \mu \mathrm{L}$ of DMSO (dimethyl sulfoxide) was added to each well of the 96-well plate to dissolve the formazan crystals formed by the viable 
cells. Finally, the plates were shaken and the absorbance of formazan dye was measured at $550 \mathrm{~nm}$ using a bench-mark microplate reader. The assay was performed in triplicate. The cytotoxic effect in each of the treatments was expressed as percentage of cell viability relative to the untreated control cells (\% control) and expressed as follows:

Cytotoxic effect $(\%)=\left(\frac{[\mathrm{OD} \text { of treated cells }] 550 \mathrm{~nm}}{[\mathrm{OD} \text { of control cells }] 550 \mathrm{~nm}}\right)$

$$
\times 100
$$

where, OD represents the optical density of the respective solution measured at $550 \mathrm{~nm}$.

\section{Characterization}

$\mathrm{X}$-ray diffraction (XRD) was employed for the crystalline phase identification of the prepared hollow spheres using $\mathrm{Cu}-\mathrm{K} \alpha$ radiation over $2 \theta$ range of $10^{\circ}-70^{\circ}$ at a scan rate of $3^{\circ} \mathrm{min}^{-1}$ and with a sampling interval of 0.05 at $40 \mathrm{~mA}$ and $40 \mathrm{kV}$ by using Bruker AXS Diffractometer D8 Powder XRD. The functional group analysis was done by Fourier transform infrared (FT-IR) spectroscopy using Perkin-Elmer Spectrum RXI instrument, within the scan range $4,000-400 \mathrm{~cm}^{-1}$. The thermogravimetric analysis (TGA) was performed using Netzsch STA 409 PC Luxx (Germany). The morphology of prepared hollow spheres were analysed by scanning electron microscopy (SEM) using SUPRA 40 Field Emission Scanning Electron Microscope (Carl Zeiss SMT AG, Germany) and transmission electron microscopy (TEM) using JEM-2100 HRTEM (JEOL, Japan) operating at $200 \mathrm{kV}$. The chemical composition of the samples were determined by X-ray photoelectron spectroscopy (XPS), carried out on PHI 5000 VersaProbe II Scanning XPS Microprobe ( $\Phi$ ULVACPHI, INC.) using monochromatic $\mathrm{AlK} \alpha$ radiations $(1,486 \mathrm{eV})$. The carbon $1 \mathrm{~s}$ peak at a standard value of $284.5 \mathrm{eV}$ has been used to calibrate the binding-energy scale for the XPS measurements. Magnetic measurements were carried out using EverCool SQUID VSM DC magnetometer (Quantam Design, USA).

\section{Results and discussion}

Crystalline phase analysis

X-ray powder diffraction (XRD) studies were carried out for all the synthesized hollow nanospheres to ascertain their crystalline phase and purity. The observed XRD patterns of the samples were shown in Fig. 1. The HT sample (i.e., undoped titania) exhibits the characteristic

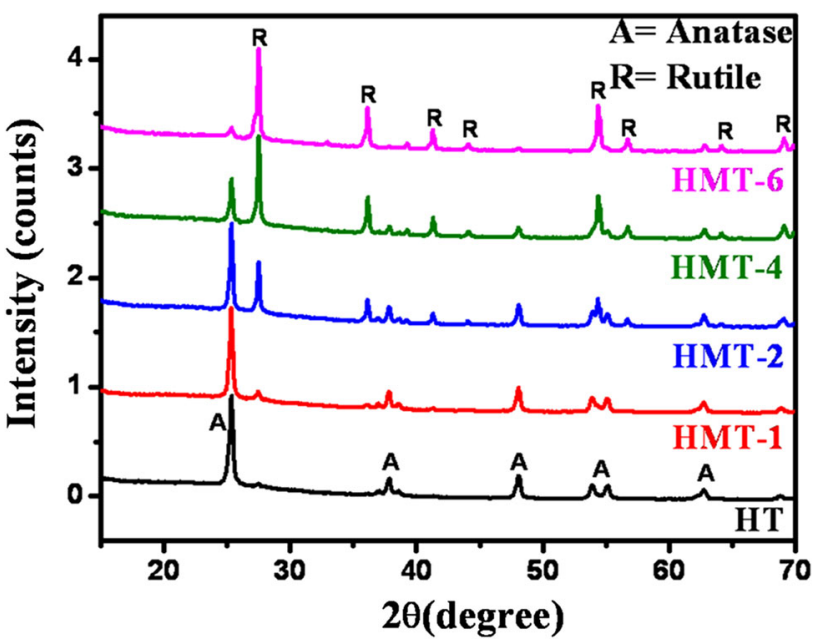

Fig. 1 XRD patterns of the prepared samples after calcining at $550{ }^{\circ} \mathrm{C}$

peaks of anatase phase of titania (JCPDS file No. 86-1157). From XRD analysis, it is apparent that with increase in the dopant (i.e., Mn) concentration, the intensity of the characteristic diffraction lines corresponding to the anatase phase of titania decreases and is gradually replaced by the characteristic diffraction lines corresponding to the rutile phase. The rutile phase (JCPDS file No. 76-0317) was eventually achieved in HMT-6, which contained 6 mol \% of $\mathrm{Mn}$ with respect to Ti. It can thus be inferred that the doped $\mathrm{Mn}$ ions gets incorporated into the titania crystal lattice and facilitates the phase transformation from anatase to rutile, when the dopant concentration is increased up to $6 \mathrm{~mol} \%$. For pure/undoped titania, the anatase to rutile phase transformation generally occurs on heating the samples at temperatures between 600 and $700{ }^{\circ} \mathrm{C}$ (Suresh et al. 1998), but it is apparent from our study that this phase transformation could be achieved at a lower temperature $\left(\sim 550{ }^{\circ} \mathrm{C}\right)$ when titania is doped with up to $6 \mathrm{~mol} \%$ of $\mathrm{Mn}$. The absence of any additional peaks in the XRD patterns of the prepared samples indicate the absence of any impurity and infers the proper doping of $\mathrm{Mn}$ ions into the crystal lattice of titania.

Functional group analysis

Fourier transform infra-red (FT-IR) spectroscopy has been carried out to analyze the functional groups present in asprepared carbon spheres, as-prepared HMT-2 as well as calcined HMT-2 samples (calcined at 450 and $550{ }^{\circ} \mathrm{C}$ ), and the spectra are depicted in Fig. 2. The observed broad band around $3,420 \mathrm{~cm}^{-1}$ in the spectrum of carbon spheres (Fig. 2a) corresponds to $\mathrm{O}-\mathrm{H}$ stretching of the carboxylic bonds (Zhang et al. 2012) formed during the dehydration process of dextrose. The band at $1,635 \mathrm{~cm}^{-1}$ can be 


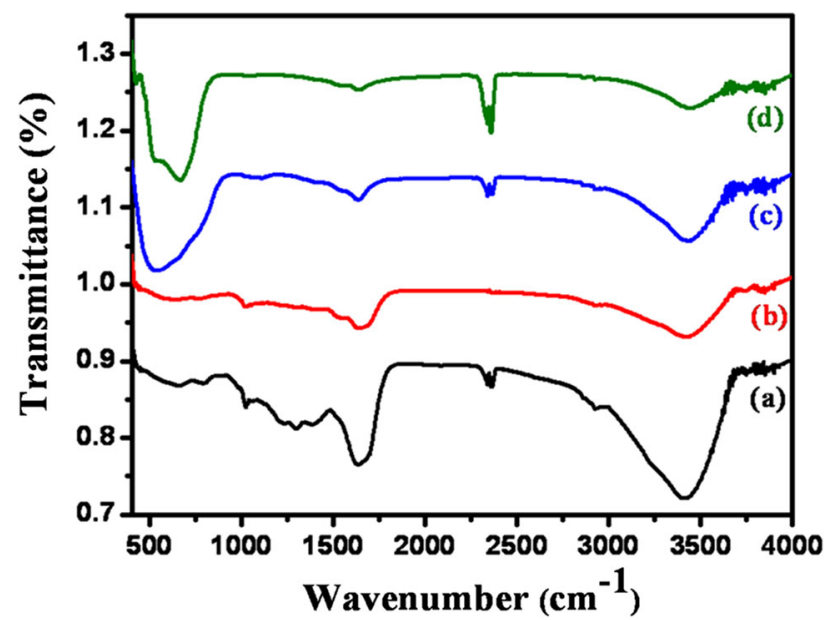

Fig. 2 FT-IR spectra of as-prepared carbon spheres (a), as-prepared HMT-2 (b), HMT-2 calcined at $450{ }^{\circ} \mathrm{C}(c)$ and $550{ }^{\circ} \mathrm{C}(d)$

attributed to $\mathrm{C}=\mathrm{C}$ stretching vibration, while the bands in the region $1,000-1,500 \mathrm{~cm}^{-1}$ indicates the existence of large amounts of residual hydroxyl groups as well as trace carbonyl groups in the prepared carbon spheres ( $\mathrm{Li}$ et al. 2010). The FT-IR spectrum of the as-prepared HMT-2 sample, depicted in Fig. 2b, shows lowering of intensity of the organic functional groups as well as the $\mathrm{O}-\mathrm{H}$ stretching band at $3,420 \mathrm{~cm}^{-1}$ present in the carbon spheres. This lowering in intensity of the functional groups present in the carbon spheres in the as-prepared HMT-2 sample, may be due to the surface coating of carbon spheres with 2 mol \% Mn-doped titania. The calcined samples of HMT-2, depicted in spectra 'c' and 'd' of Fig. 2, shows further weakening and eventual disappearance of the bands associated with the carbon spheres when the calcination temperature was increased from 450 to $550{ }^{\circ} \mathrm{C}$. This could be due to the complete removal of the carbon spheres from HMT-2 samples during calcination. However, the calcined samples of HMT-2 depicted a new broad band centred at around $530 \mathrm{~cm}^{-1}$, which became prominent with increase in the calcination temperature from 450 to $550{ }^{\circ} \mathrm{C}$. This band could be attributed to the Ti-O bending vibrations, which could be associated with the crystallization of titania phase in the samples upon calcination.

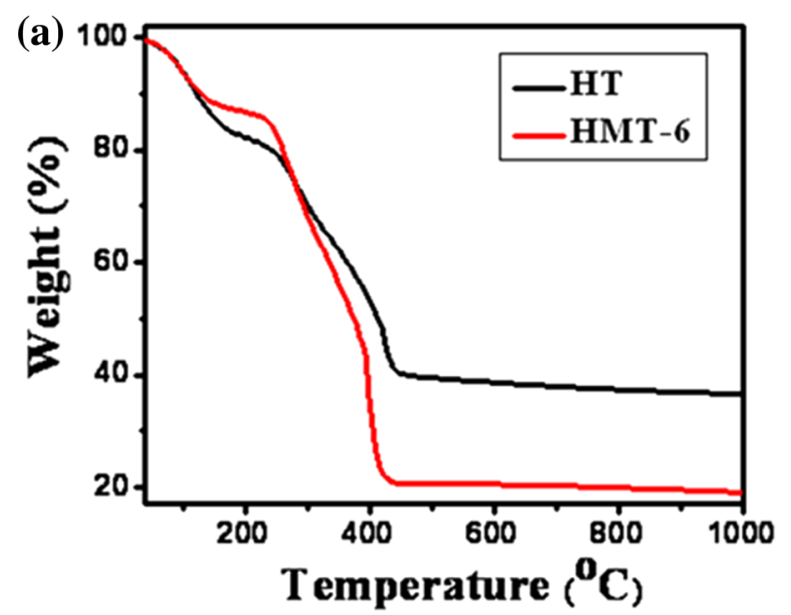

(b)

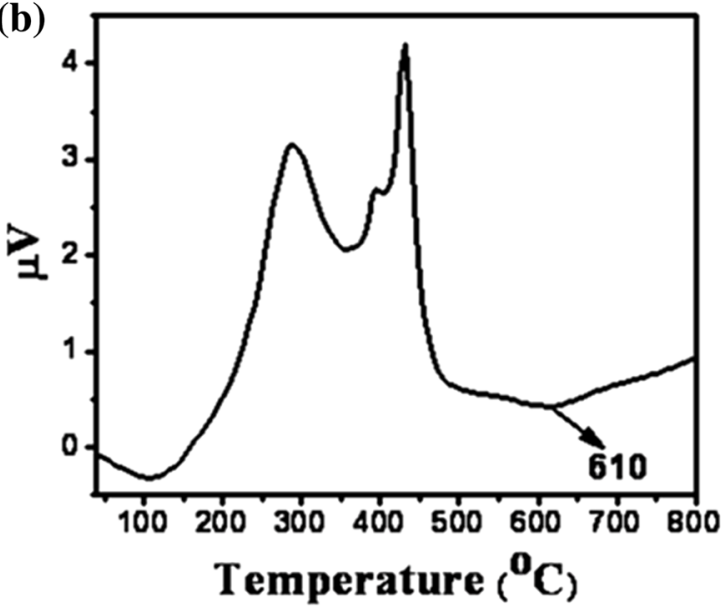

(c)

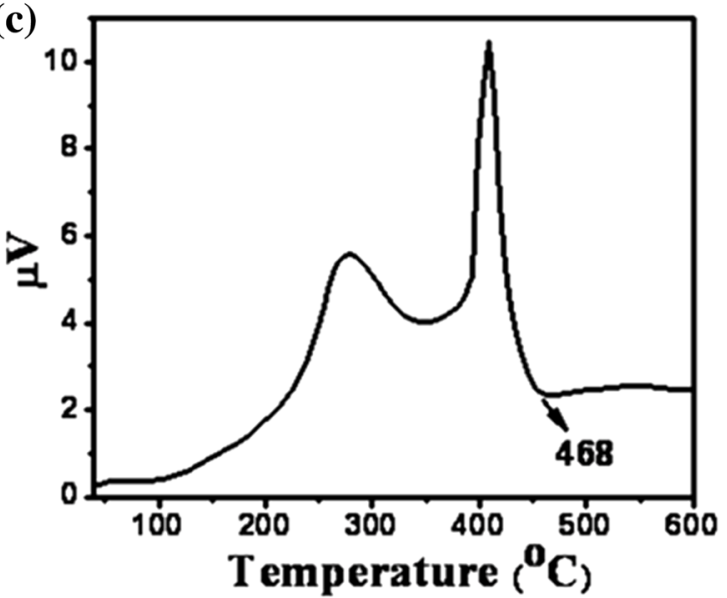

Fig. 3 TGA curves for as-prepared samples of HT and HMT-6 (a), DTA curves for HT (b) and HMT-6 (c) 
Thermal studies

TGA-DTA studies were carried out for the as-prepared samples of HT and HMT- 6 for determining the calcination temperature required for the complete removal of the carbon core and the formation of crystalline titania. The thermal studies were performed by heating the samples in alumina crucibles from room temperature to $1,000{ }^{\circ} \mathrm{C}$ in air at a heating rate of $10^{\circ} \mathrm{C} / \mathrm{min}$. The TGA curves for both the samples, shown in Fig. 3a, reveal four regions of significant weight loss below $450{ }^{\circ} \mathrm{C}$. The first weight loss from room temperature $\left(29{ }^{\circ} \mathrm{C}\right)$ to $140{ }^{\circ} \mathrm{C}$ corresponds to the loss of physically adsorbed water, while the second weight loss from 140 to around $235^{\circ} \mathrm{C}$ attributes to the loss of crystallization water. The third weight loss between 235 and $395{ }^{\circ} \mathrm{C}$ represents the loss of the carbon core through combustion and the fourth weight loss from 395 to $445^{\circ} \mathrm{C}$ corresponds to the crystallization of amorphous state to anatase phase of titania for both the samples (i.e., HT and
HMT-6). Beyond $450{ }^{\circ} \mathrm{C}$, the TGA curves for both the samples show no significant weight loss, indicating the formation of thermally stable titania phase. The DTA curves for the two samples, represented in Fig. 3b, c, shows an endothermic peak at about $100{ }^{\circ} \mathrm{C}$ for both the samples, corresponding to the removal of water moieties from the samples, while two exothermic peaks centred at 290 and $430{ }^{\circ} \mathrm{C}$ for HT and at 280 and $409{ }^{\circ} \mathrm{C}$ for HMT-6 samples are observed. The first exothermic peak corresponds to the removal of carbon core, while the second peak represents the crystallization of amorphous state to anatase phase of titania. A third exothermic peak starts appearing at 610 and $468{ }^{\circ} \mathrm{C}$ for HT and HMT-6, respectively, that represent the temperature for phase transformation from anatase to rutile phase of titania. It is visible that on doping titania with $6 \mathrm{~mol} \% \mathrm{Mn}$, the phase transformation temperature is lowered from $610{ }^{\circ} \mathrm{C}$ in $\mathrm{HT}$ (undoped titania) to $468^{\circ} \mathrm{C}$ in HMT-6 samples, consistent with the XRD patterns of the samples analysed after calcining at $550{ }^{\circ} \mathrm{C}$.
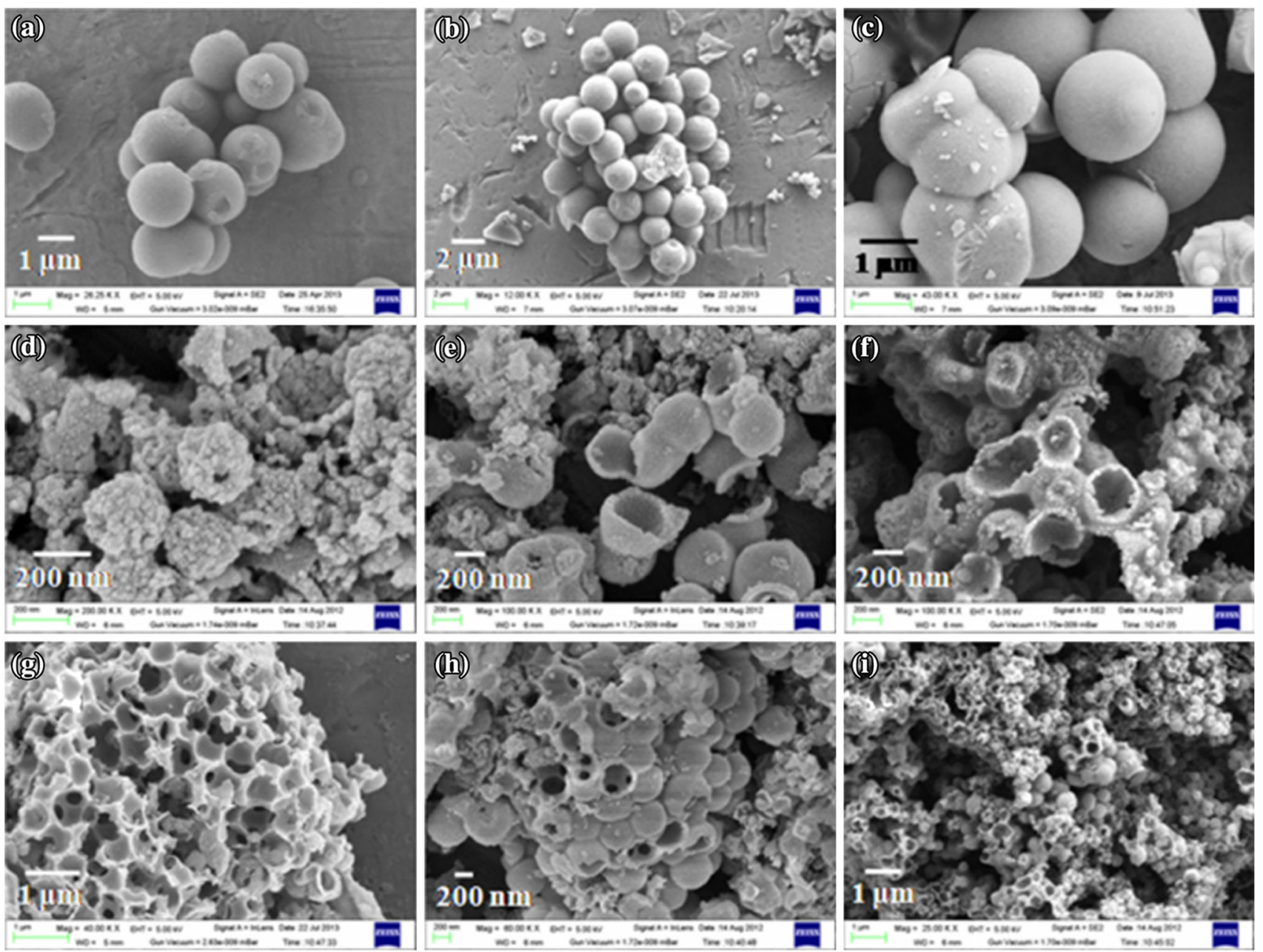

Fig. 4 SEM images of as-prepared carbon spheres (a), HT (b), and HMT-1 (c), calcined HMT-1 (d), broken spheres of HMT-1 (e) and HMT-2 (f), hollow three-dimensional network structure of HT (g), HMT-1 (h), and HMT-2 (i)

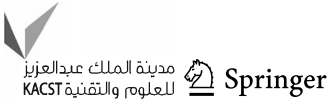


Morphological analysis

The morphological characteristic of the as-prepared carbon spheres as well as the as-prepared and calcined Mndoped samples of titania were determined by scanning electron microscopic (SEM) studies. Spherical shape with smooth surface and almost uniform diameters of the as-prepared carbon spheres is evident from their SEM images shown in Fig. 4a. The as-prepared samples of HT and HMT-1, obtained on surface coating of carbon spheres with titania and $1 \mathrm{~mol} \%$ Mn-doped titania, respectively, also possesses smooth surface and spherical shapes as apparent from their SEM images shown in Fig. $4 \mathrm{~b}$, c, respectively. After calcining the samples at $550{ }^{\circ} \mathrm{C}$, the surfaces become a bit rough due to the contraction of titania precursor associated with the removal
Fig. 5 TEM images of HT (a) and HMT-1 (b) (inset their corresponding SAED patterns)
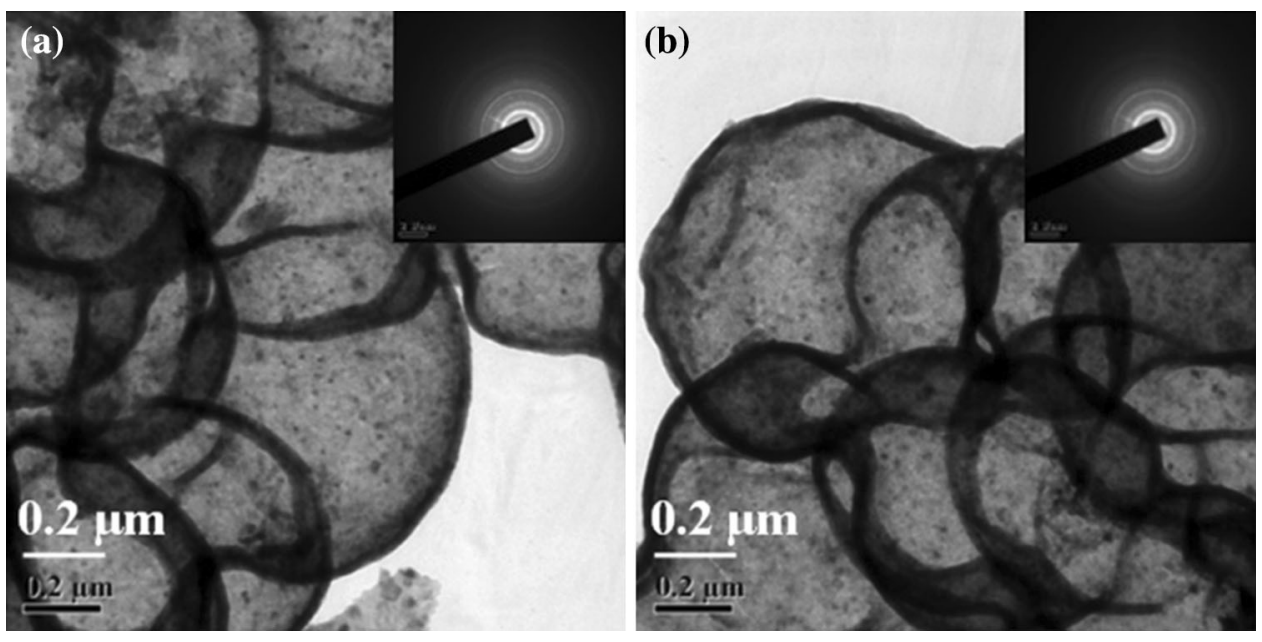
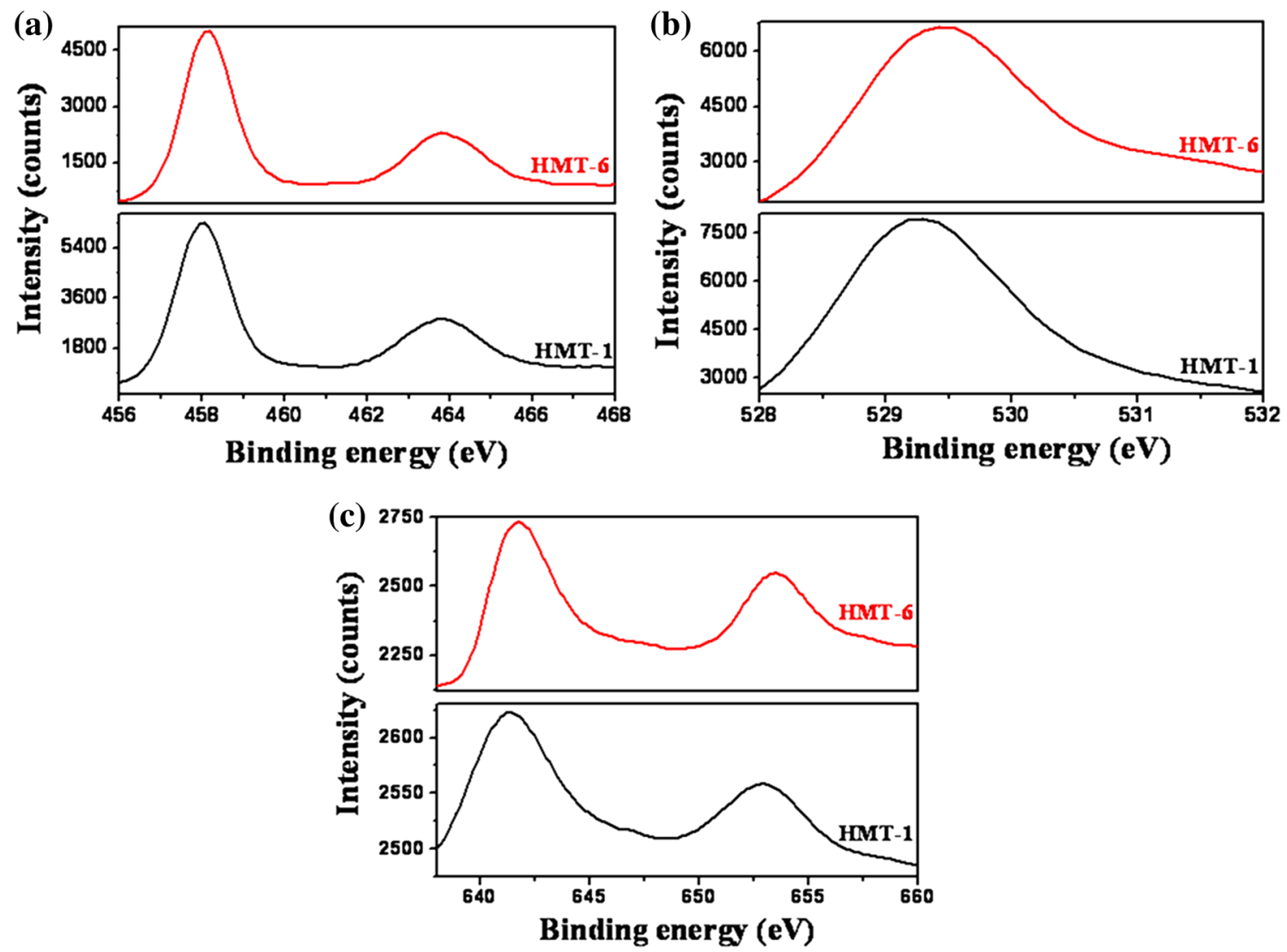

Fig. 6 XPS spectra of Ti 2p core levels (a), O 1s core levels (b), and Mn 2p core levels (c) for HMT-1 and HMT-6 
of carbon core, possessing a diameter of about $400 \mathrm{~nm}$, as represented by the SEM image of HMT-1 in Fig. 4d. The hollow nature of the calcined samples with wall thickness of around $40 \mathrm{~nm}$ may be inferred from the ruptured spheres seen in the SEM images of HMT-1 and HMT-2 in Fig. 4e, f, respectively. In addition, the SEM images shown in Fig. $4 \mathrm{~g}-\mathrm{i}$ infers that the hollow spheres get interlinked on calcination to generate a three-dimentional network structure.

Spherical shape and hollow nature of the calcined (at $550{ }^{\circ} \mathrm{C}$ ) undoped and $\mathrm{Mn}$-doped titania samples are also evident from transmission electron microscopic (TEM) studies. The bright field TEM images of calcined HT and HMT-1 samples are shown in Fig. 5 as typical
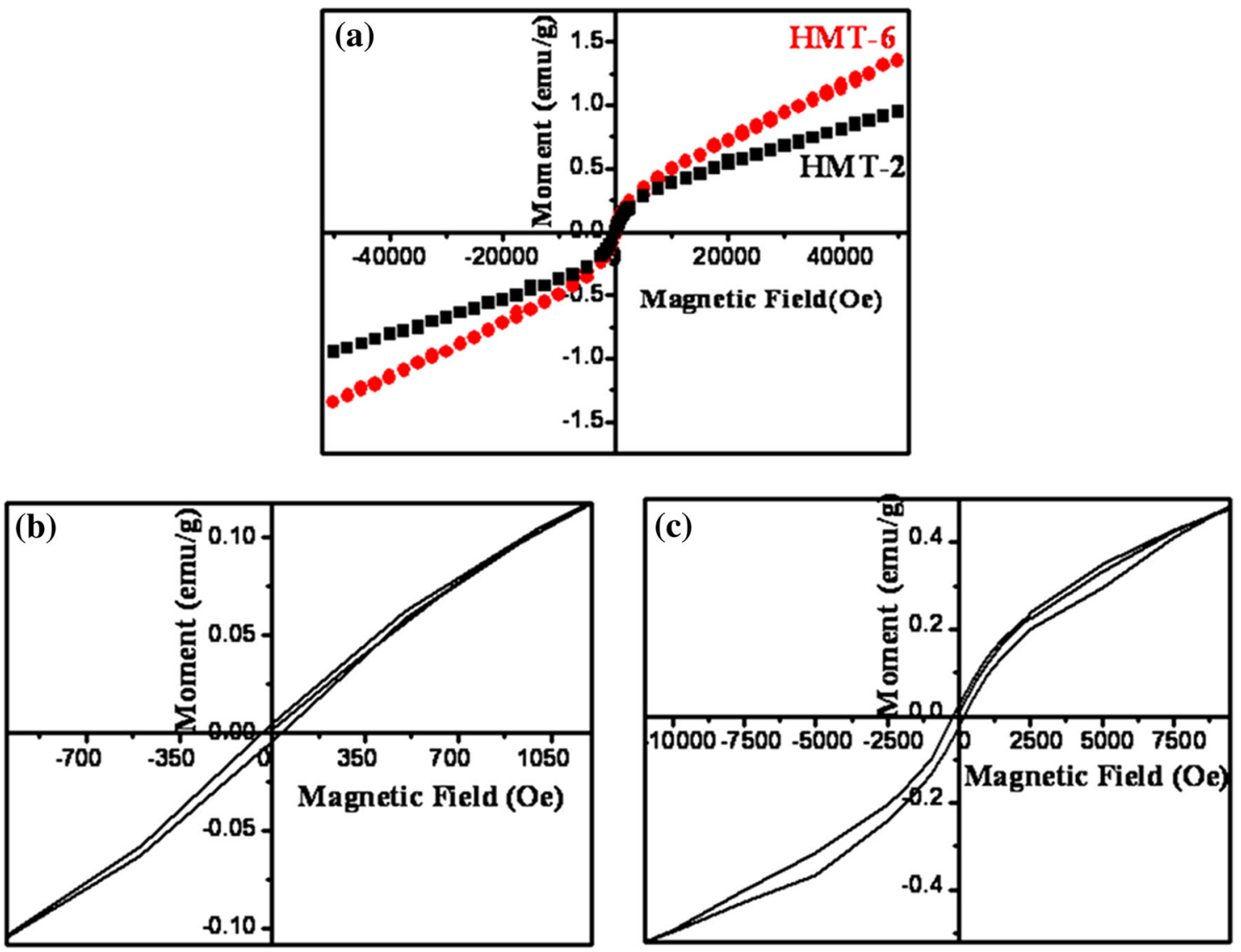

Fig. $7 \mathrm{M}(\mathrm{H})$ curves for HMT-6 and HMT-2 (a), hysteresis loops for HMT-2 (b) and HMT-6 (c)
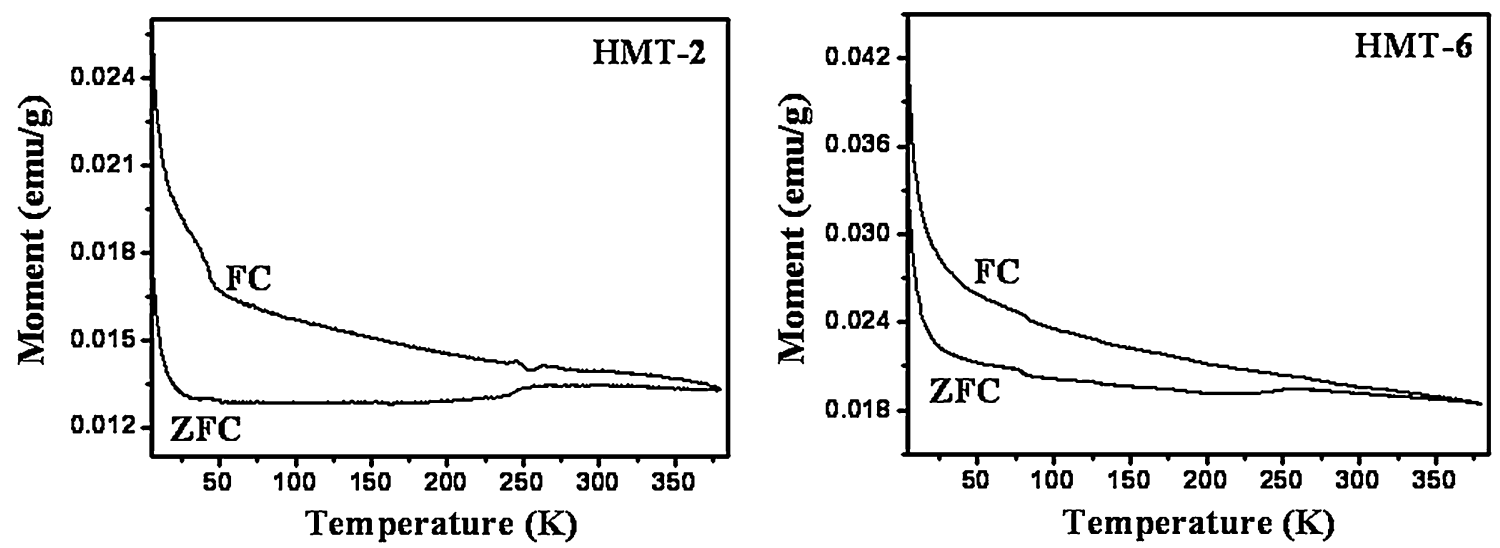

Fig. 8 ZFC- FC curves for HMT-2 and HMT-6 
representatives, while their corresponding SAED patterns are shown as inset in their respective bright field TEM images in Fig. 5a, b, respectively.

\section{XPS analysis}

X-ray photoelectron spectroscopy (XPS) was employed on HMT-1 and HMT-6 samples to determine their chemical structure and the results were shown in Fig. 6. The XPS spectra of $\mathrm{Ti} 2 \mathrm{p}$ core levels (Fig. 6a) for HMT-1 shows binding energy peaks for Ti $2 p_{3 / 2}$ and Ti $2 p_{1 / 2}$ at 458.05 and $463.79 \mathrm{eV}$, respectively, while the binding energy peaks of $\mathrm{Ti} 2 \mathrm{p}_{3 / 2}$ and $\mathrm{Ti} 2 \mathrm{p}_{1 / 2}$ peaks for HMT-6 were observed at 458.1 and $463.8 \mathrm{eV}$, respectively. The corresponding Ti $2 p$ doublet separation for HMT-1 and HMT-6 was 5.72 and $5.7 \mathrm{eV}$, respectively. The position of the $\mathrm{Ti}$ $2 p$ doublet peaks and the separation value between 5.7 and $6 \mathrm{eV}$ indicates the presence of $\mathrm{Ti}^{4+}$ state in the prepared spheres (Liu et al. 2007).

The XPS spectra of O 1s core level for HMT-1 and HMT-6 are shown in Fig. 6b. The binding energy peak for O 1s was observed at 529.29 and $529.4 \mathrm{eV}$ for HMT-1 and HMT-6, respectively. These binding energy values correspond to the normal lattice sites in the titania $\left(\mathrm{TiO}_{2}\right)$ structure with oxygen in $\mathrm{O}^{2-}$ state in the hollow spheres.

The XPS spectra of Mn $2 p$ core levels for the samples were shown in Fig. 6c. The binding energy peaks corresponding to $\mathrm{Mn} 2 \mathrm{p}_{3 / 2}$ and $\mathrm{Mn} 2 \mathrm{p}_{1 / 2}$ peaks were observed at 641.3 and $652.9 \mathrm{eV}$, respectively, for HMT-1 and 641.7 and $653.4 \mathrm{eV}$, respectively, for HMT-6 with a doublet separation of 11.6 and $11.7 \mathrm{eV}$ for HMT-1 and HMT-6, respectively. The peak position and doublet separation values for the samples correspond to the presence of $\mathrm{Mn}^{2+}$ state in the prepared spheres. In addition, it was observed that the binding energy peaks shows a positive shift for all the three elements in HMT-6 as compared to HMT-1, which indicates the more positive charged surface of HMT6 which is due to the high concentration of dopant (i.e., Mn) ions in HMT-6 as compared to HMT-1.

\section{Magnetic measurements}

Magnetic measurements of the prepared HMT-2 and HMT6 samples were performed with superconducting quantum interference device (SQUID). The field-dependent magnetization $\mathrm{M}(\mathrm{H})$ curves of the samples at room temperature $(300 \mathrm{~K})$, shown in Fig. $7 \mathrm{a}$, reveals that the saturation magnetization of HMT-6 is higher as compared to HMT-2, suggesting the increase in magnetization with increase in $\mathrm{Mn}$ content. The coercive field and saturation magnetization for HMT-2 and HMT- 6 were found to be 34.7 Oe; $0.037 \mathrm{emu} / \mathrm{g}$ and $183.7 \mathrm{Oe} ; 0.048 \mathrm{emu} / \mathrm{g}$, respectively. The hysteresis loops of the samples at $300 \mathrm{~K}$, shown in Fig. 7b, c, shows that both the samples exhibit room temperature ferromagnetism. The observed ferromagnetism in the samples (at $300 \mathrm{~K}$ ) is due to the incorporation of Mn ions into the titania crystal lattice. Any possible contribution from manganese oxides and/or Ti/Mn binary oxides can be ruled out, since they are ferromagnetic only at $100 \mathrm{~K}$ or less (Tian et al. 2008).

The temperature dependent magnetization in HMT-2 and HMT-6 have been shown by the zero-field-cooled (ZFC) and field-cooled (FC) magnetization curves carried out between 5 and $380 \mathrm{~K}$ under an applied field of 100 Oe. The ZFC and FC magnetization curves for HMT-2 and HMT-6, shown in Fig. 8, reveals the absence of blocking temperature for both the samples. In addition, the divergence of the FC and ZFC curves at temperatures well above $300 \mathrm{~K}$ indicates that the $\mathrm{T}_{\mathrm{C}}$ (Curie temperature) of both the samples is above $300 \mathrm{~K}$ and both the samples exhibit ferromagnetism at room temperature.

\section{Cytotoxicity studies}

To determine the safety and toxicological level of the prepared nanospheres for intracellular applications, the cytotoxicity of the prepared spheres were examined by MTT assay using human breast cancer cell line (MDA-MB 231) treated with various concentrations of the spheres. The plot of concentration versus percentage of cell viability (shown in Fig. 9) for the various nanospheres reveals that the cell viability is more than $50 \%$ (i.e., half maximal inhibitory concentration, $\mathrm{IC}_{50}$ ) even when their dosage is as high as $200 \mu \mathrm{g} / \mathrm{mL}$. The $\mathrm{IC}_{50}$ value for HT, HMT-1, HMT-2, HMT4, and HMT-6 was found to be 293.9, 246.7, 280, 202.3, and $243.4 \mu \mathrm{g} / \mathrm{mL}$, respectively. For all the prepared nanospheres, the $\mathrm{IC}_{50}$, values indicate that they are all biocompatible and could be used for intracellular applications.

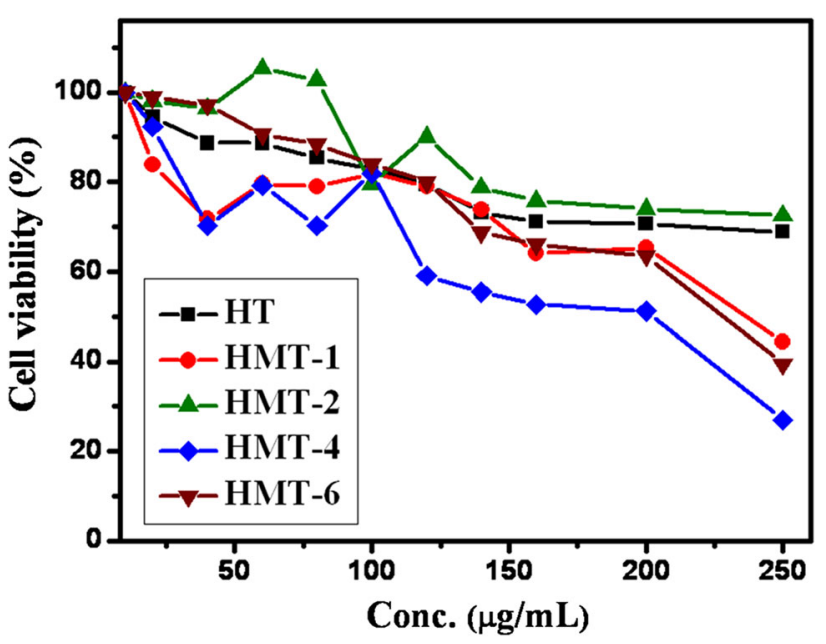

Fig. 9 MTT assay for the prepared samples 


\section{Control}

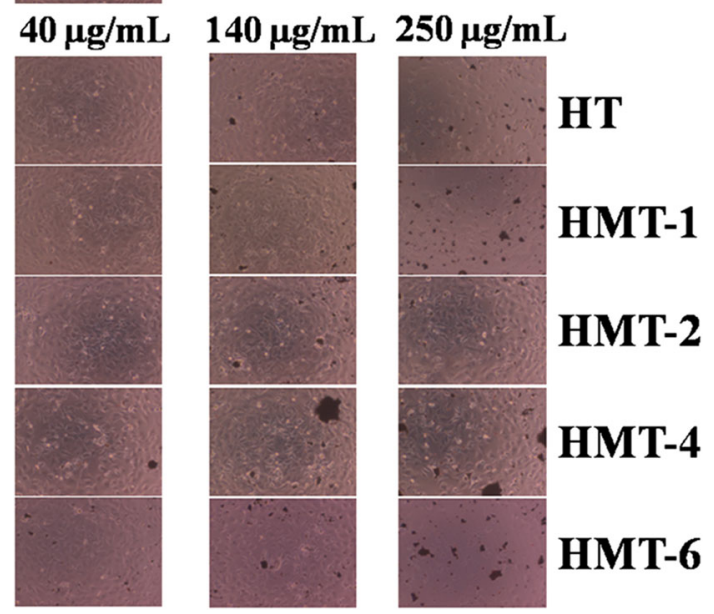

Fig. 10 Optical microscopic images of the cells after $48 \mathrm{~h}$ of incubation with the prepared hollow spheres

The observations from MTT assay was further substantiated through optical microscopic images of the cell lines taken after $48 \mathrm{~h}$ of incubation with different concentrations of the prepared nanospheres. The optical microscopic images displaying the amount of viable cells left on the wells of the 96-well plate is shown in Fig. 10.

\section{Conclusions}

In conclusion, Mn-doped titania hollow spheres have been successfully prepared through sacrificial core templating method using carbon spheres as the sacrificial core templates. The hollow spherical structures of the calcined samples, with diameters of about $400 \mathrm{~nm}$ and wall thickness of $40 \mathrm{~nm}$, were confirmed through SEM and TEM studies. XRD studies revealed the anatase phase for undoped titania sample which got transformed into the rutile phase with increase in the dopant (i.e., Mn) concentration at temperature as low as $550{ }^{\circ} \mathrm{C}$. XPS studies shows the presence of $\mathrm{Ti}^{4+}$ state for $\mathrm{Ti}, \mathrm{O}^{2-}$ state for $\mathrm{O}$, and $\mathrm{Mn}^{2+}$ state for Mn. Magnetic studies confirmed the presence of room temperature ferromagnetism in the $\mathrm{Mn}$ doped samples. Further, the samples were found to be biocompatible with $\mathrm{IC}_{50}$ values higher than $200 \mu \mathrm{g} / \mathrm{mL}$ for all the samples, thereby validating their applicability for different biomedical applications.

Acknowledgments The authors are thankful to Prof. Mahitosh Mandal, School of Medical Science and Technology, IIT Kharagpur India, for helping with the cytotoxicity studies. The authors would also like to acknowledge the DST-FIST funded XPS facility at the Department of Physics and Meteorology, IIT Kharagpur India and University Grants Commission (UGC) New Delhi, India for financial support.

Open Access This article is distributed under the terms of the Creative Commons Attribution License which permits any use, distribution, and reproduction in any medium, provided the original author(s) and the source are credited.

\section{References}

Andronescu E, Ficai A, Georgiana M, Mitran V, Sonmez M, Ficai D, Ion R, Cimpean A (2013) Collagen-hydroxyapatite/cisplatin drug delivery systems for locoregional treatment of bone cancer. Technol Cancer Res T 12:275-284

Ao YH, Xu JJ, Zhang SH, Fu DG (2010) A one-pot method to prepare $\mathrm{N}$-doped titania hollow spheres with high photocatalytic activity under visible light. Appl Surf Sci 256:2754-2758

Aw MS, Addai-Mensah J, Losic D (2012) A multi-drug delivery system with sequential release using titania nanotube arrays. Chem Commun 48:3348-3350

Batra B, Lata S, Rana JS, Pundir CS (2013) Construction of an amperometric bilirubin biosensor based on covalent immobilization of bilirubin oxidase onto zirconia coated silica nanoparticles/chitosan hybrid film. Biosens Bioelectron 44:64-69

Bernal C, Illanes A, Wilson L (2014) Heterofunctional hydrophilic hydrophobic porous silica as support for multipoint covalent immobilization of lipases: application to lactulose palmitate synthesis. Langmuir 30:3557-3566

Daumann LJ, Larrabee JA, Ollis D, Schenk G, Gahan LR (2014) Immobilization of the enzyme GpdQ on magnetite nanoparticles for organophosphate pesticide bioremediation. J Inorg Biochem 131:1-7

Dorniani D, Hussein MZ, Kura AU, Fakurazi S, Shaari AH, Ahmad Z (2013) Preparation and characterization of 6-mercaptopurinecoated magnetite nanoparticles as a drug delivery system. Drug Des Dev Ther 7:1015-1026

Gulati K, Aw MS, Findlay D, Losic D (2012) Local drug delivery to the bone by drug-releasing implants: perspectives of nanoengineered titania nanotube arrays. Ther Deliv 3:857-873

Hou JW, Dong GX, Ye Y, Chen V (2014) Laccase immobilization on titania nanoparticles and titania-functionalized membranes. J Membrane Sci 452:229-240

Kim G, Hong LY, Jung J, Kim DP, Kim H, Kim IJ, Kim JR, Ree M (2010) The biocompatability of mesoporous inorganic-organic hybrid resin films with ionic and hydrophilic characteristics. Biomaterials 31:2517-2525

Li XZ, Chen F, Lu XW, Ni CY, Zhao XB, Chen ZG (2010) Layer-bylayer synthesis of hollow spherical $\mathrm{CeO}_{2}$ templated by carbon spheres. J Porous Mat 17:297-303

Liang HB, Li XF, Xiong L, Huang SM (2013) Synthesis and characterization of titania nanoparticles functionalized with hyperbranched polymer via self-condensing vinyl polymerization. Synth React Inorg M 43:1034-1039

Liu GX, Shan FK, Lee WJ, Shin BC (2007) Growth temperature dependence of $\mathrm{TiO}_{2}$ thin films prepared by using plasmaenhanced atomic layer deposition method. J Korean Phys Soc 50:1827-1832

Liu MJ, Ishida Y, Ebina Y, Sasaki T, Aida T (2013) Photolatently modulable hydrogels using unilamellar titania nanosheets as photocatalytic crosslinkers. Nat Commun 4:2029

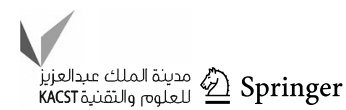


Mascolo MC (2013) Synthesis of wide spectrum of mesoporous titania materials by forced co-hydrolysis of $\mathrm{Zr}-\mathrm{Ti}$ alkoxides. Micropor Mesopor Mat 181:160-165

Pandikumar A, Ramaraj R (2013) Photocatalytic reduction of hexavalent chromium at gold nanoparticles modified titania nanotubes. Mater Chem Phys 141:629-635

Pang AY, Xia LC, Luo HY, Li YF, Wei MD (2013) Highly efficient indoline dyes co-sensitized solar cells composed of titania nanorods. Electrochim Acta 94:92-97

Saponjic ZV, Dimitrijevic NM, Poluektov OG, Chen LX, Wasinger E, Welp U, Tiede DM, Zuo XB, Rajh T (2006) Charge separation and surface reconstruction: $\mathrm{a} \mathrm{Mn}^{2+}$ doping study. J Phys Chem B 110:25441-25450

Sun J, Wu JM (2013) A comparative study on photocatalytic activity of titania nanowires subjected to high-temperature calcination and low-temperature $\mathrm{HCl}$ treatment. Sci Adv Mater 5:549-556

Suresh C, Biju V, Mukundan P, Warrier KGK (1998) Anatase to rutile transformation in sol-gel titania by modification of precursor. Polyhedron 17:3131-3135

Tang SH, Huang XQ, Chen XL, Zheng NF (2010) Hollow mesoporous zirconia nanocapsules for drug delivery. Adv Funct Mater 20:2442-2447

Tian ZM, Yuan SL, Wang YQ, He JH, Yin SY, Liu KL, Yuan SJ, Liu $\mathrm{L}$ (2008) Magnetic studies on Mn-doped $\mathrm{TiO}_{2}$ bulk samples. J Phys D Appl Phys 41:055006
Venkatesan P, Puvvada N, Dash R, Kumar BNP, Sarkar D, Azab B, Pathak A, Kundu SC, Fisher PB, Mandal M (2011) The potential of celecoxib-loaded hydroxyapatite-chitosan nanocomposite for the treatment of colon cancer. Biomaterials 32:3794-3806

Wang B, Zhang JJ, Pan ZY, Tao XQ, Wang HS (2009) A novel hydrogen peroxide sensor based on the direct electron transfer of horseradish peroxidase immobilized on silica-hydroxyapatite hybrid film. Biosens Bioelectron 24:1141-1145

Wu KCW, Yamauchi Y, Hong CY, Yang YH, Liang YH, Funatsu T, Tsunoda M (2011) Biocompatible, surface functionalized mesoporous titania nanoparticles for intracellular imaging and anticancer drug delivery. Chem Commun 47:5232-5234

Xu JP, Lin YB, Lu ZH, Liu XC, Lu ZL, Wang JF, Zou WQ, Lv LY, Zhang FM, Du YW (2006) Enhanced ferromagnetism in Mndoped $\mathrm{TiO}_{2}$ films during the structural phase transition. Solid State Commun 140:514-518

Xu JH, Gao FP, Li LL, Ma HL, Fan YS, Liu W, Guo SS, Zhao XZ, Wang H (2013) Gelatin-mesoporous silica nanoparticles as matrix metalloproteinases-degradable drug delivery systems in vivo. Micropor Mesopor Mat 182:165-172

Zhang M, Yang H, Liu YN, Sun XD, Zhang DK, Xue DF (2012) First identification of primary nanoparticles in the aggregation of HMF. Nanoscale Res Lett 7:38

Zhao CX, Middelberg APJ (2013) One-step fabrication of titania hollow spheres by controlled interfacial reaction in a dropletbased microfluidic system. Microfluid Nanofluid 14:703-709 